\title{
Robust automated cardiac arrhythmia detection in ECG beat signals
}

\author{
Victor Hugo C. de Albuquerque ${ }^{1}$ Thiago M. Nunes ${ }^{2}$ - Danillo R. Pereira ${ }^{3}$. \\ Eduardo José da S. $\mathrm{Luz}^{4} \cdot$ David Menotti $^{5} \cdot$ João P. Papa $^{3} \cdot$ João Manuel R. S. Tavares $^{6}$
}

Received: 16 December 2015/ Accepted: 6 July 2016/Published online: 19 July 2016

(C) The Natural Computing Applications Forum 2016

\begin{abstract}
Nowadays, millions of people are affected by heart diseases worldwide, whereas a considerable amount of them could be aided through an electrocardiogram (ECG) trace analysis, which involves the study of arrhythmia impacts on electrocardiogram patterns. In this work, we carried out the task of automatic arrhythmia detection in ECG patterns by means of supervised machine learning techniques, being the main contribution of this paper to introduce the optimum-path forest (OPF) classifier to this context. We compared six distance metrics, six feature extraction algorithms and three classifiers in two variations of the same dataset, being the performance of the techniques compared in terms of effectiveness and efficiency. Although OPF revealed a higher skill on generalizing data, the support vector machines (SVM)-based classifier presented the highest accuracy. However, OPF
\end{abstract}

João Manuel R. S. Tavares

tavares@fe.up.pt

Victor Hugo C. de Albuquerque

victor.albuquerque@unifor.br

Thiago M. Nunes

tmnun@hotmail.com

Danillo R. Pereira

dpereira@ic.unicamp.br

Eduardo José da S. Luz

eduluz@gmail.com

David Menotti

menotti@inf.ufpr.br

João P. Papa

papa@fc.unesp.br

1 Laboratório de Bioinformática, Programa de Pós-Graduação em Informática Aplicada, Universidade de Fortaleza,

Fortaleza, CE, Brazil shown to be more efficient than SVM in terms of the computational time for both training and test phases.

Keywords ECG heart beats - Electrophysiological signals · Cardiac dysrhythmia classification - Feature extraction · Pattern recognition - Optimum-path forest

\section{Introduction}

The automatic detection and classification of arrhythmias in electrocardiography-based signals (ECG) has been widely studied in the last years in order to aid the diagnose of heart diseases. One way to perform this type of test is to conduct a long-time recording of the cardiac activity of an individual in his/her normal routine in order to obtain a

Centro de Ciências Tecnológicas Universidade de Fortaleza, Fortaleza, CE, Brazil

3 Departamento de Ciência da Computação, Universidade Estadual Paulista, Bauru, São Paulo, Brazil

4 Departamento de Computação, Universidade Federal de Ouro Preto, Ouro Prêto, MG, Brazil

5 Departamento de Informática, Universidade Federal do Paraná, Curitiba, PR, Brazil

6 Instituto de Ciência e Inovação em Engenharia Mecânica e Engenharia Industrial, Departamento de Engenharia Mecânica, Faculdade de Engenharia, Universidade do Porto, Porto, Portugal 
reasonable amount of information about the individual's heartbeats. However, the posterior task of analyzing such data may be tiresome and more prone to errors when interpreted by human beings, since there is a huge amount of information to be processed.

In order to cope with such problem, several works have been carried out arrhythmia classification in EEG signals by means of machine learning-oriented techniques $[1,5,14,15,18]$. However, regardless of the classification algorithm used, some processing steps are crucial to design a reasonable approach to detect arrhythmia. The quality of classification when dealing with ECG signals is directly dependent on the preprocessing phase, which aims at filtering noise frequencies that might interfere with ECG signal [21]. After preprocessing, it is required to detect and segment each heartbeat of the ECG signal. In order to perform this task, an important step is the detection of the QRS complex (three deflections from ECG signal), specifically the $R$ wave, since most part of the techniques for the detection and segmentation of heartbeats are based on the location of such deflection. Because of the steep angular coefficient and amplitude of the $R$ wave, the QRS complex becomes more obvious than any other part of the ECG signal, being easier to be detected for later segmentation.

The final step is the classification of ECG signals, which is usually accomplished in a supervised fashion. Support vector machines (SVMs) [1, 7-9, 13, 27, 29, 31] and artificial neural networks (ANNs) [6, 10, 12, 14, 20, 23, 28, 30, 32, 33] are among the most used machine learning techniques for this purpose. Other approaches such as linear discriminant analysis [5] and a hybridization of support vector machines and artificial neural networks [11] are also applied for heartbeat classification. However, one of the main shortcomings related to the aforementioned pattern recognition techniques concern with their parameters, which need to be fine-tuned prior to their application over the unseen samples (test set). SVMs are known due to their good skills on generalizing over test samples, but with the cost of having a high computational burden when learning the statistics of the training data, since each different kernel has its own parameters to be set up. ANNs are usually very fast for classifying samples, but its training step may be trapped in local optima, as well as it is not straightforward to choose a proper neural architecture.

Based on such assumptions, Papa et al. [25, 26] proposed the optimum-path forest (OPF) classifier, which is a framework for designing classifiers based on graph partitions, being the samples (feature vectors) encoded by graph nodes and connected to each other by means of a predefined adjacency relation. A set of key nodes (prototypes) competes among themselves in order to conquer the remaining nodes offering to them optimum-path costs. This competition process generates a set of optimum-path trees rooted at each prototype node, meaning that a sample of a given tree is more strongly connected to its root than to any other in the forest.

The OPF classifier has gained considerable attention in the last years, since it has some advantages over traditional classifiers: (1) it is free of hard-to-calibrate control parameters; (2) it does not assume any shape/separability of the feature space; (3) it runs the training phase usually much faster; and (4) it can take decisions based on global criteria. However, to the best of our knowledge, the OPF classifier has never been employed to aid the diagnosis of arrhythmias in heart rate by means of ECG signals so far. Therefore, the main contribution of this paper is to evaluate OPF effectiveness in ECG-based arrhythmia classification, being its results compared against some stateof-the-art pattern recognition techniques in terms of accuracy, computational time, sensitivity and specificity. Finally, another contribution of this work is to assess the performance of six different feature extraction methods in the aforementioned context, mainly: the approaches proposed by Chazal et al. [5], Güler and Übeyli [10], Song et al. [29], Yu and Chen [32], You and Chou [33], and Ye et al. [31].

\section{Methodology}

In this section, we describe the methodology employed in this work. Initially, the MIT-BIH (Massachusetts Institute of Technology_Beth Israel Hospital Boston) arrhythmia database [19] is described addressing considerations of ANSI/AAMI standard EC57 [3], which standardizes the evaluation of computational tools for the classification of cardiac arrhythmia datasets. After that, the feature extraction techniques used to generate the feature vectors are then described, followed by the description of the statistical parameters used to evaluate the performance of the classifiers under comparison.

\subsection{MIT-BIH arrhythmia database}

The MIT-BIH arrhythmia database is composed of signals from electrocardiography exams, being widely used to evaluate the performance of algorithms concerning the task of detecting arrhythmias [22]. The data consist of 48 records, $30 \mathrm{~min}$ long, taken from $24 \mathrm{~h}$ of ECG acquisition, being the samples obtained from two different channels. The signals were acquired from 47 patients between 1975 and 1979 at the laboratory of Arrhythmia Boston's Beth Israel Hospital, which are aged between 23 and 89 years of which 22 females and 25 males. The analog records were digitized according to a sampling rate of $360 \mathrm{~Hz}$, and the heartbeats marked and manually classified by experts in 15 classes regarding the type of arrhythmia. The types of arrhythmia identified in the database are indicated in Table 1. 
Table 1 Types of heartbeats presented in the MIT-BIH database grouped according to AAMI Standard

\begin{tabular}{lll}
\hline AAMI class & MIT-BIH original class & Type of beat \\
\hline Normal $(N)$ & $N$ & Normal beat \\
& $L$ & Left bundle branch block beat \\
& $R$ & Right bundle branch block beat \\
& $e$ & Atrial escape beat \\
& $j$ & Nodal (junctional) escape beat \\
Supraventricular ectopic beat $(S)$ & $A$ & Atrial premature beat \\
& $a$ & Aberrated atrial premature beat \\
& $J$ & Nodal (junctional) premature beat \\
Ventricular ectopic beat $(V)$ & $S$ & Supraventricular premature beat \\
Fusion beat $(F)$ & $V$ & Premature ventricular contraction \\
Unknown beat $(Q)$ & $E$ & Ventricular escape beat \\
& $F$ & Fusion of ventricular and normal beat \\
& $/$ & Paced beat \\
& $f$ & Fusion of paced and normal beat \\
& $Q$ & Unclassifiable beat \\
\hline
\end{tabular}
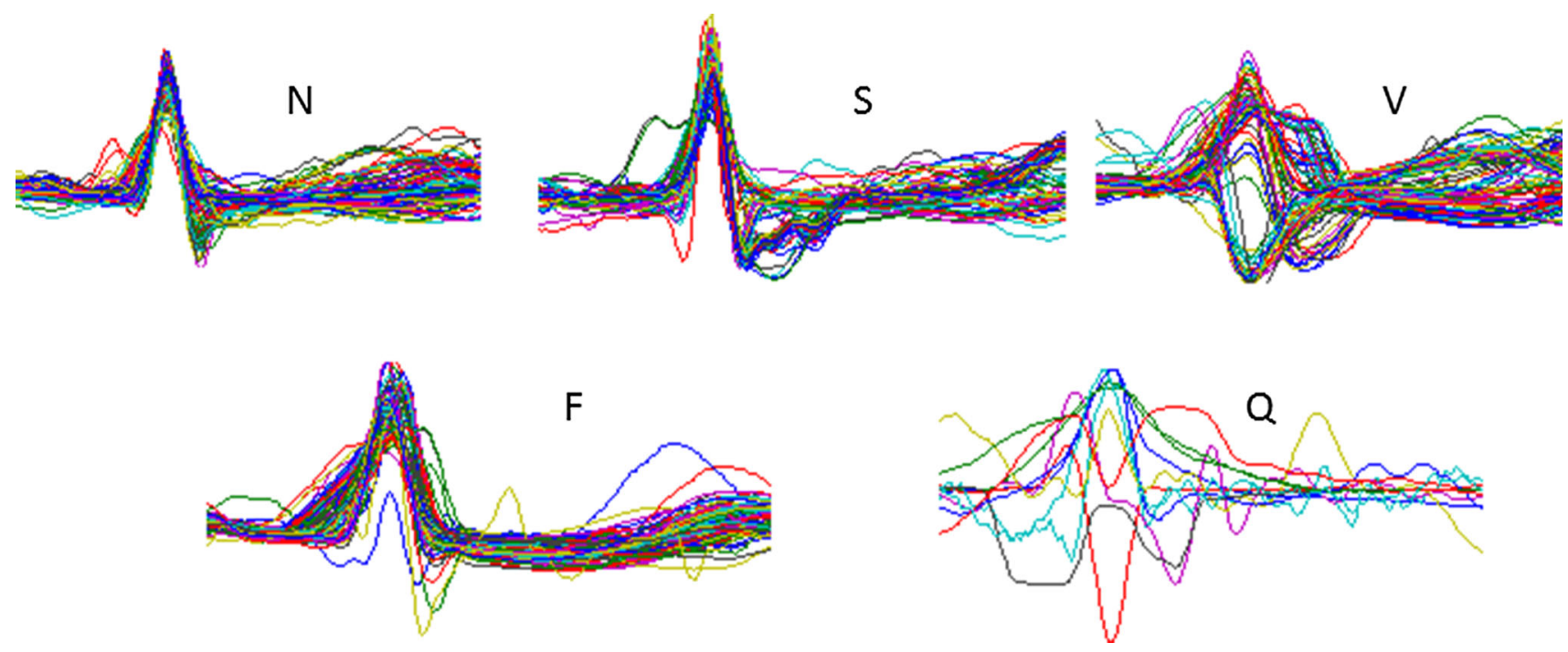

Fig. 1 MIT-BIH heartbeat signals grouped according to [3]

Since the detection and segmentation of beats in ECG signals is not the main goal of this work, we have employed precomputed annotations of $R$ waves provided by the database in order to accomplish the signal segmentation. In addition, 4 records derived from patients that make use of pacemakers that were discarded, following the recommendation of ANSI/AAMI standard EC57 [3], which also recommends to group the 15 classes reported in the database's annotations into 5 classes (Table 1). Figure 1 depicts some ECG signals for each class, being class $Q$ represented by 10 signals, and the remaining ones represented by 100 signals. The signals were randomly picked up from the database.

\subsection{Training and test set}

The database was partitioned into two sets of records in order to separate the patients in training and testing groups. The composition of both sets was based on the study of Chazal et al. [5], which proposed to separate the patients by balancing each heartbeat class, as presented in Table 2 . Besides the division of heartbeats into 5 classes as defined in [3], it was also considered the classification of heartbeats proposed by Llamedo and Martínez [15], which divided the 5 classes proposed in [3] into three main classes: $N, S$ and $V$. Classes $F$ and $Q$, which are less significant, were added to class $V$. 
Table 2 Composition of the training and test sets according to Chazal et al. [5]

\begin{tabular}{ll}
\hline Set & Records \\
\hline Training & $101,106,108,109,112,114,115,116,118,119,122,124,201,203,205,207,208,209,215,220,223$ e 230 \\
Test & $100,103,105,11,113,117,121,123,200,202,210,212,213,214,219,221,222,228,231,232,233$ e 234 \\
\hline
\end{tabular}

Table 3 Description of the experimental datasets according to AAMI classes [3]

\begin{tabular}{llrllllll}
\hline Dataset & Feature extraction & $n_{f}$ & \multicolumn{2}{l}{ Hearbeat class } & & \multirow{2}{*}{$T_{b}$} \\
\cline { 5 - 8 } & & & $N$ & $S$ & $V$ & $F$ & $Q$ & \\
\hline Training & & & & & & & & \\
A & {$[5]$} & 155 & 45,747 & 940 & 3777 & 415 & 8 & 50,887 \\
B & {$[10]$} & 19 & 45,845 & 943 & 3788 & 415 & 8 & 50,999 \\
C & {$[29]$} & 21 & 45,825 & 943 & 3788 & 414 & 8 & 50,978 \\
D & {$[32]$} & 13 & 45,844 & 943 & 3788 & 415 & 8 & 50,998 \\
E & {$[33]$} & 31 & 45,511 & 929 & 3770 & 412 & 8 & 50,630 \\
F & {$[31]$} & 100 & 45,844 & 943 & 3788 & 415 & 8 & 50,998 \\
Test & & & & & & & & \\
A & {$[5]$} & 155 & 44,181 & 1786 & 3218 & 388 & 7 & 49580 \\
B & {$[10]$} & 19 & 44,238 & 1836 & 3221 & 388 & 7 & 49690 \\
C & {$[29]$} & 21 & 44,218 & 1836 & 3219 & 388 & 7 & 49,668 \\
D & {$[32]$} & 13 & 44,238 & 1836 & 3221 & 388 & 7 & 49,690 \\
E & {$[33]$} & 31 & 43,905 & 1823 & 3197 & 388 & 7 & 49,320 \\
F & {$[31]$} & 100 & 44238 & 1836 & 3221 & 388 & 7 & 49,690 \\
\hline
\end{tabular}

\subsection{Feature extraction}

Six feature extraction approaches (associated with Dataset A-F) were chosen based on the work of Luz and Menotti [16], which performed a comparison among some of the most used approaches for such purpose, mainly: Discrete Wavelet Transform (DWT), Independent Component Analysis (ICA), Principal Component Analysis (PCA), as well as information about RR range/interspace, which is the distance between peaks of two successive $R$ waves in an ECG signal. For each dataset, the following methods were considered in this work:

- Dataset $\mathrm{A}-$ morphology of the signal and RR range [5];

- Dataset B-DWT [10];

- Dataset C-DWT [29];

- Dataset D-DWT, RR range and signal energy [32];

- Dataset E-DWT, ICA and RR range [33] and

- Dataset F-DWT, ICA, PCA and RR range [31].

The distribution of heartbeats by class and feature extraction approach considering the division of classes proposed by [3] is shown in Table 3, while Table 4 displays the same information considering the distribution into 3 classes proposed by [15]. In this Table $T_{b}$ and $n_{f}$ stand for the number of heartbeats of the set and the number of features extracted by each technique, respectively. One can noticed
Table 4 Description of the experimental datasets according to [15]

\begin{tabular}{|c|c|c|c|c|c|c|}
\hline \multirow[t]{2}{*}{ Dataset } & \multirow[t]{2}{*}{ Method } & \multirow[b]{2}{*}{$n_{f}$} & \multicolumn{3}{|c|}{ Heartbeat class } & \multirow[t]{2}{*}{$T_{b}$} \\
\hline & & & $N$ & SVEB & VEB & \\
\hline \multicolumn{7}{|l|}{ Train } \\
\hline A & [5] & 155 & 45,747 & 940 & 4200 & 50,887 \\
\hline B & [10] & 19 & 45,845 & 943 & 4211 & 50,999 \\
\hline $\mathrm{C}$ & [29] & 21 & 45,825 & 943 & 4210 & 50,978 \\
\hline D & [32] & 13 & 45,844 & 943 & 4211 & 50,998 \\
\hline E & [33] & 31 & 45,511 & 929 & 4190 & 50,630 \\
\hline $\mathrm{F}$ & [31] & 100 & 45,844 & 943 & 4211 & 50,998 \\
\hline \multicolumn{7}{|l|}{ Test } \\
\hline A & [5] & 155 & 44,181 & 1786 & 3613 & 49580 \\
\hline B & [10] & 19 & 44,238 & 1836 & 3616 & 49,690 \\
\hline $\mathrm{C}$ & [29] & 21 & 4,4218 & 1836 & 3614 & 49,668 \\
\hline D & {$[32]$} & 13 & 44,238 & 1836 & 3616 & 49,690 \\
\hline $\mathrm{E}$ & {$[33]$} & 31 & 43,905 & 1823 & 3592 & 49,320 \\
\hline $\mathrm{F}$ & [31] & 100 & 44,238 & 1836 & 3616 & 49,690 \\
\hline
\end{tabular}

the variation in the number of beats among the methods concerns with the feature extraction techniques that usually do not allow using the entire database. Samples located at the extremities of the signal, for instance, do not contain enough neighboring samples/segments to perform the proper feature extraction. 


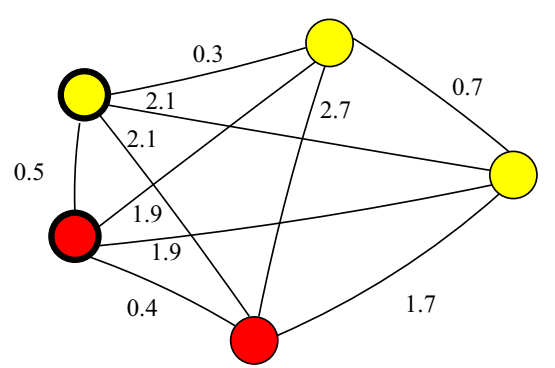

(a)

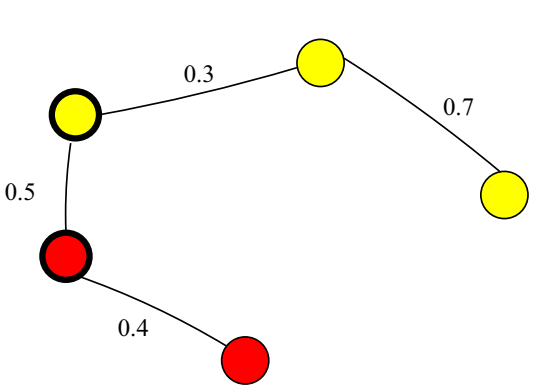

(b)

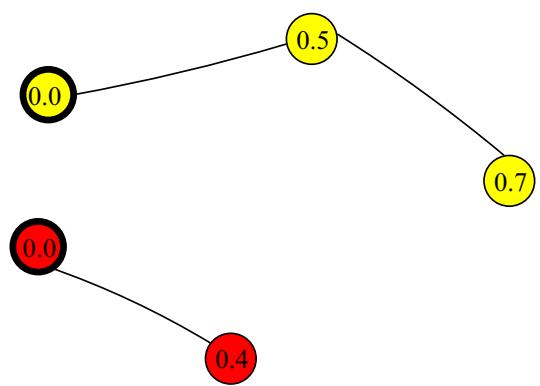

(c)

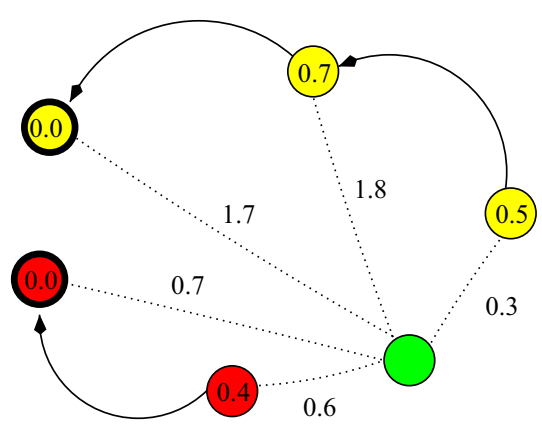

(d)

Fig. 2 a In the training step the training set is modeled as a complete graph, b a minimum spanning tree over the training set is computed (prototypes are highlighted), $\mathbf{c}$ optimum-path forest over the training

\subsection{Optimum-path forest classifier}

Let $\mathcal{D}=\mathcal{D}_{1} \cup \mathcal{D}_{2}$ be a $\lambda$-labeled dataset, where $\mathcal{D}_{1}$ and $\mathcal{D}_{2}$ denote the training and test sets, respectively. Let $\mathcal{S} \subset \mathcal{D}_{1}$ be a set of prototypes of all classes (i.e., the key samples that best represent each samples class). The complete graph $\left(\mathcal{D}_{1}, A\right)$ is composed of nodes that represent samples in $\mathcal{D}_{1}$, and any pair of samples defines an edge in $A=\mathcal{D}_{1} \times \mathcal{D}_{1}$ (Fig. 2a). ${ }^{1}$ Additionally, let $\left.\pi_{s}=<s_{1}, s_{2}, \ldots, s_{n}, s\right\rangle$ be a path with terminus at node $s \in \mathcal{D}_{1}$.

Roughly speaking, the OPF classifier contains two distinct phases, being the first one employed for training purposes, and the latter used to assess the robustness of the classifier designed in the previous phase. The training phase aims at building the optimum-path forest, and the test step classifies each test node individually, i.e. ,they are added to the training set for classification purposes only, and further removed.

\subsubsection{Training step}

$\mathcal{S}^{*}$ is an optimum set of prototypes when the OPF algorithm minimizes the classification errors for every $s \in \mathcal{D}_{1}$. Such set $S^{*}$ can be found by the theoretical association between the minimum spanning tree (MST) and the optimum-path tree

\footnotetext{
1 The edges are weighted by the distance between their corresponding samples/nodes.
}

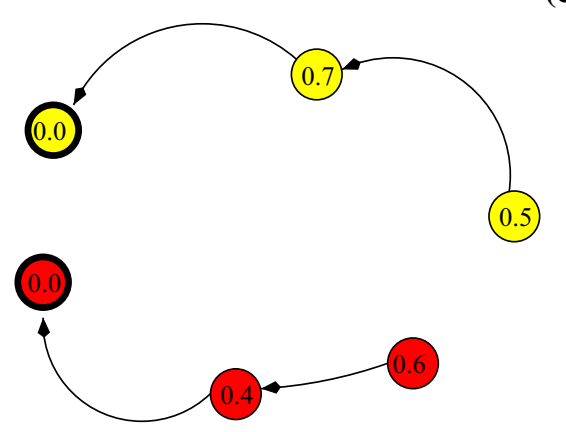

(e)

set, $\mathbf{d}$ classification process of a test sample (in green), and e test sample classification (color figure online)

for $f_{\max }$ [2]. Briefly, the training is the process of finding the $S^{*}$ and an OPF classifier rooted at $S^{*}$. The MST in the complete graph $\left(\mathcal{D}_{1}, A\right)$ (Fig. $\left.2 \mathrm{~b}\right)$ is represented by a connected acyclic graph whose nodes are all samples of $\mathcal{D}_{1}$, and the edges are undirected and weighted by the distances $d$ between two adjacent samples. Every pair of samples is connected by a single path, which is minimum according to $f_{\max }$. Hence, the minimum spanning tree contains one optimum-path tree for any selected root node.

The optimum prototypes are the closest nodes of the MST with different labels in $\mathcal{D}_{1}$ (i.e., samples that fall in the frontier of the classes, as highlighted in Fig. 2b). Removing the edges between different classes, their adjacent nodes become prototypes in $S^{*}$. The OPF algorithm can define an optimum-path forest with minimum classification errors in $\mathcal{D}_{1}$ (Fig. 2c).

Soon after finding prototypes, the OPF algorithm is used, which essentially aims at minimizing the cost of every training sample. Such cost is computed using the $f_{\max }$ path-cost function, given by:

$f_{\max }(\langle s\rangle)=\left\{\begin{array}{cl}0 & \text { if } \mathrm{s} \in \mathrm{S} \\ +\infty & \text { otherwise },\end{array}\right.$

$f_{\max }\left(\pi_{s} \cdot\langle s, t\rangle\right)=\max \left\{f_{\max }\left(\pi_{s}\right), d(s, t)\right\}$,

where $\langle s\rangle$ is a trivial path, $\langle s, t\rangle$ is the arc between the adjacent nodes $s$ and $t$ such that $s, t \in \mathcal{D}_{1}, d(s, t)$ denotes the distance between nodes $s$ and $t$, and $\pi_{s} \cdot\langle s, t\rangle$, is the 
concatenation of path $\pi_{s}$ with the arc $\langle s, t\rangle$. One can note that $f_{\max }\left(\pi_{s}\right)$ computes the maximum distance between adjacent samples in $\pi_{s}$ when $\pi_{s}$ is not a trivial path. Roughly speaking, the OPF algorithm aims at minimizing $f_{\max }\left(\pi_{t}\right), \quad \forall t \in \mathcal{D}_{1}$.

\subsubsection{Classification step}

For any node $t \in \mathcal{D}_{2}$, we consider all edges connecting $t$ with samples $s \in \mathcal{D}_{1}$, as though $t$ were part of the training graph (Fig. 2d). Considering all possible paths from $S^{*}$ to $t$, OPF finds the optimum path $P^{*}(t)$ from $S^{*}$ and labels $t$ with the class $\lambda(R(t))$ of its most strongly connected prototype $R(t) \in S^{*}$ (Fig. 2e). This path can be identified incrementally evaluating the optimum cost $C(t)$ :

$$
C(t)=\min \{\max \{C(s), d(s, t)\}\}, \forall s \in D_{1} .
$$

Let the node $s^{*} \in \mathcal{D}_{1}$ be the one that satisfies Eq. (2) (i.e., the $P(t)$ in the optimum path $P^{*}(t)$ ). Given that $L\left(s^{*}\right)=\lambda(R(t))$, the classification simply assigns $L\left(s^{*}\right)$ as the class of $t$. An error occurs when $L\left(s^{*}\right) \neq \lambda(t)$.

\section{Results and discussion}

In this section, we present the experimental results concerning the effectiveness and efficiency of each pair classifier/feature extraction technique employed in this work. First of all, the OPF classifier is evaluated considering six distance metrics: Euclidean, Chi-square, Manhattan, Chisquared and squared Bray-Curtis. After that, a comparison among OPF with the best metrics, support vector machines with radial basis function (SVM-RBF) and a Bayesian classifier (BC), is then presented.

\subsection{Experimental analysis of optimum-path forest}

In this section, we evaluate the performance and the computational time of the OPF classifier using six distance metrics. $^{2}$ The evaluation is performed considering the classification according to five [3] and three classes [15].

\subsubsection{Five-class problem}

Here, we present the results considering the experimental dataset divided into five classes. Table 5 displays the recognition rates obtained by OPF using each distance metric ${ }^{3}$ in the datasets defined by each feature extraction approach.

\footnotetext{
${ }^{2}$ For such purpose, we used the LibOPF library [24].

3 The recognition rates were computed using the standard formula, i.e., the ratio of the number of correct classifications by the number of database samples and $\mathrm{H}$ the harmonic mean between sensitivity and specificity.
}

Table 5 OPF accuracy considering 5 classes

\begin{tabular}{llll}
\hline Dataset & \multicolumn{2}{l}{ Distance metric } \\
\cline { 2 - 4 } & Euclidean (\%) & Chi-square (\%) & Manhattan (\%) \\
\hline A & 80.68 & 83.26 & 77.57 \\
B & 79.63 & 88.80 & 79.43 \\
C & 81.25 & 87.60 & 84.46 \\
D & 90.70 & 89.12 & $\mathbf{9 1 . 2 1}$ \\
E & 86.54 & 89.05 & 86.47 \\
F & 89.12 & 85.28 & 90.39 \\
\hline Dataset & Distance metric & \\
\cline { 2 - 4 } & Canberra (\%) & Squared Chi-squared (\%) & Bray-Curtis (\%) \\
\hline A & 77.93 & 76.14 & 79.81 \\
B & 80.51 & 80.61 & 87.69 \\
C & 84.90 & 82.63 & 76.55 \\
D & 90.88 & 90.75 & 88.90 \\
E & 86.53 & 86.62 & 81.79 \\
F & 86.60 & 85.70 & 78.41 \\
\hline
\end{tabular}

The most accurate result is indicated in bold

We can observe that OPF with Manhattan distance obtained the best recognition rate with dataset $\mathrm{D}$ $(91.21 \%)$, and that is approximately $0.35 \%$ higher than the second best result obtained with the Canberra distance metric $(90.88 \%)$, as well as $0.5 \%$ higher than the result obtained with the squared Chi-squared metric $(90.75 \%)$. Additionally, the results using dataset $\mathrm{D}$ were the best for all employed distances, suggesting that the method proposed by $\mathrm{Yu}$ and Chen [32] might be a good feature extractor to be used together with OPF. In addition to the recognition rate, we also computed the sensitivity (Se) and specificity (Sp), as well as the harmonic mean $(H)$ of these two parameters (Table 6).

The best values of $H$ considering class $N$ were obtained using Canberra (0.78) and squared Chi-squared (0.78) distances and feature extractor $\mathrm{C}$. The combination of squared Chi-squared metric and extractor $\mathrm{C}$ resulted in the best value of $H$ for class $S$ (0.60). In regard to classes $V$ and $F$, Euclidean distance has provided the best results with feature extractor F. As to class $Q$, OPF did not classify any sample properly due to the following main factors: the nonconcentrated distribution of samples from that class and the low representation of samples in the training and test sets ( $\sim 0.00015 \%$ of the total number of samples).

However, a high recognition rate not always reflects a satisfactory performance in terms of classes separation, once that only class $N$ (patient without cardiac arrhythmia) represents $\approx 90 \%$ of all dataset. For instance, let us consider the case of Chi-square metric, which presented the best accuracy rates for feature extractor B (Table 5). The 
Table 6 Specificity, sensitivity and their harmonic mean considering the OPF classifier and the AAMI five-class categorization

\begin{tabular}{|c|c|c|c|c|c|c|}
\hline \multirow[t]{2}{*}{ Metrics } & \multirow[t]{2}{*}{ Dataset } & \multicolumn{5}{|l|}{ Heartbeat classes } \\
\hline & & $\begin{array}{l}N \\
\mathrm{H}-\mathrm{Se}-\mathrm{Sp}\end{array}$ & $\begin{array}{l}S \\
\mathrm{H}-\mathrm{Se}-\mathrm{Sp}\end{array}$ & $\begin{array}{l}V \\
\mathrm{H}-\mathrm{Se}-\mathrm{Sp}\end{array}$ & $\begin{array}{l}F \\
\mathrm{H}-\mathrm{Se}-\mathrm{Sp}\end{array}$ & $\begin{array}{l}Q \\
\mathrm{H}-\mathrm{Se}-\mathrm{Sp}\end{array}$ \\
\hline \multirow[t]{6}{*}{ Euclidean } & A & $066-085-054$ & $002-001-097$ & $084-078-091$ & $\mathbf{0 5 5}-038-097$ & $000-000-100$ \\
\hline & $\mathrm{B}$ & $050-086-035$ & $005-002-097$ & $056-041-090$ & $001-001-098$ & $000-000-100$ \\
\hline & $\mathrm{C}$ & $074-085-066$ & $031-018-095$ & $084-078-091$ & $014-007-097$ & $000-000-100$ \\
\hline & $\mathrm{D}$ & $073-096-059$ & $030-018-099$ & $084-075-097$ & 007-004-099 & $000-000-100$ \\
\hline & $\mathrm{E}$ & $065-092-050$ & $006-003-098$ & $076-062-097$ & $029-017-097$ & $000-000-100$ \\
\hline & $\mathrm{F}$ & $074-093-062$ & $022-012-099$ & 091-086-097 & $031-018-097$ & $000-000-100$ \\
\hline \multirow[t]{6}{*}{ Chi-square } & A & $017-093-009$ & $003-001-099$ & $015-008-095$ & $005-002-099$ & $000-000-100$ \\
\hline & $\mathrm{B}$ & $002-100-001$ & $000-000-100$ & $000-000-100$ & $001-001-100$ & $000-000-100$ \\
\hline & $\mathrm{C}$ & $015-098-008$ & $002-001-100$ & 017-009-098 & $000-000-100$ & $000-000-100$ \\
\hline & $\mathrm{D}$ & $004-100-002$ & $000-000-100$ & $004-002-100$ & $000-000-100$ & $000-000-100$ \\
\hline & $\mathrm{E}$ & $004-100-002$ & $000-000-100$ & $004-002-100$ & $000-000-100$ & $000-000-100$ \\
\hline & $\mathrm{F}$ & $012-095-006$ & $002-001-099$ & $011-006-097$ & $001-001-100$ & $000-000-100$ \\
\hline \multirow[t]{6}{*}{ Manhattan } & A & $066-081-056$ & $003-002-095$ & $086-082-090$ & $051-035-096$ & $000-000-100$ \\
\hline & $\mathrm{B}$ & $046-087-031$ & $006-003-097$ & $051-035-090$ & $001-000-099$ & $000-000-100$ \\
\hline & $\mathrm{C}$ & $076-088-066$ & $031-018-097$ & $085-078-093$ & $013-007-098$ & $000-000-100$ \\
\hline & $\mathrm{D}$ & $071-096-056$ & $023-013-099$ & $085-075-098$ & $008-004-099$ & $000-000-100$ \\
\hline & $\mathrm{E}$ & $064-092-048$ & $006-003-098$ & $075-061-097$ & $023-013-097$ & $000-000-100$ \\
\hline & $\mathrm{F}$ & $072-095-058$ & $010-006-099$ & $090-083-098$ & $034-021-098$ & $000-000-100$ \\
\hline \multirow[t]{6}{*}{ Canberra } & A & $071-081-063$ & $047-031-098$ & $080-073-087$ & $025-015-096$ & $000-000-100$ \\
\hline & $\mathrm{B}$ & $042-088-028$ & $005-003-098$ & $045-029-091$ & $002-001-099$ & $000-000-100$ \\
\hline & $\mathrm{C}$ & 078-088-071 & $056-039-096$ & $084-077-093$ & $016-009-099$ & $000-000-100$ \\
\hline & $\mathrm{D}$ & $071-096-057$ & $026-015-099$ & $085-075-098$ & $011-006-099$ & $000-000-100$ \\
\hline & $\mathrm{E}$ & $064-092-049$ & $007-004-098$ & $076-062-097$ & $024-014-097$ & $000-000-100$ \\
\hline & $\mathrm{F}$ & $064-092-049$ & $007-004-099$ & $083-071-098$ & $009-005-095$ & $000-000-100$ \\
\hline \multirow[t]{6}{*}{ Squared Chi-square } & A & $065-080-056$ & $006-003-097$ & $081-076-086$ & $037-023-097$ & $000-000-100$ \\
\hline & $\mathrm{B}$ & $048-088-033$ & $004-002-098$ & $053-037-091$ & $001-001-098$ & $000-000-100$ \\
\hline & $\mathrm{C}$ & 078 $-085-072$ & 060-044-095 & $085-078-093$ & $022-012-097$ & $000-000-100$ \\
\hline & $\mathrm{D}$ & $073-096-060$ & $032-019-099$ & $085-075-097$ & 009-005-099 & $000-000-100$ \\
\hline & $\mathrm{E}$ & $065-092-050$ & $006-003-098$ & $076-062-097$ & $027-016-097$ & $000-000-100$ \\
\hline & $\mathrm{F}$ & $069-090-056$ & $020-011-099$ & 087-079-098 & $008-004-093$ & $000-000-100$ \\
\hline \multirow[t]{6}{*}{ Bray-Curtis } & A & $051-086-036$ & $018-010-098$ & $057-041-090$ & 016-009-098 & $000-000-100$ \\
\hline & B & $005-098-002$ & $000-000-100$ & $000-000-098$ & $000-000-100$ & $000-000-100$ \\
\hline & $\mathrm{C}$ & $055-083-041$ & $008-004-096$ & $057-043-088$ & $001-000-099$ & $000-000-100$ \\
\hline & $\mathrm{D}$ & $002-100-001$ & $001-000-100$ & $000-000-100$ & $000-000-100$ & $000-000-100$ \\
\hline & $\mathrm{E}$ & $036-090-022$ & $005-003-098$ & $044-028-096$ & $006-003-096$ & $000-000-100$ \\
\hline & $\mathrm{F}$ & $053-085-039$ & $003-002-096$ & $054-038-091$ & $021-012-098$ & $000-000-100$ \\
\hline
\end{tabular}

The best values for the harmonic mean are indicated in bold. Notice the $H$, Se and Sp values are not divided by 100 due to the lack of space

good results of such metric did not lead us to a satisfactory performance in terms of classes separation, since it presented low values for sensitivity and specificity for all classes, except for class $N$. This is due to the misclassification of most samples of classes $S, V, F$ and $Q$, as belonging to class $N$, leading to a low harmonic mean $(2 \%)$. In order to clarify this, the confusion matrix related to feature extractor B and squared Chi-square metric was built, Table 7 . From the data obtained, one can verify that the dataset is dominated by class $N$, which clearly influ- 
Table 7 Confusion matrix obtained for Chi-square and feature extractor B

\begin{tabular}{lrrrrr}
\hline & \multicolumn{2}{l}{ True class } & & & \\
\cline { 2 - 6 } & \multicolumn{1}{c}{$N$} & $S$ & $V$ & \multicolumn{1}{l}{$F$} & $Q$ \\
\hline \multicolumn{2}{l}{ Predicted class } \\
$N$ & 44,115 & 1834 & 3209 & 350 & 7 \\
$S$ & 27 & 0 & 2 & 4 & 0 \\
$V$ & 76 & 0 & 7 & 32 & 0 \\
$F$ & 19 & 2 & 3 & 2 & 0 \\
$Q$ & 1 & 0 & 0 & 0 & 0 \\
\hline
\end{tabular}

enced all other classes. This can be confirmed by analyzing the results obtained for classes $S, V, F$ and $Q$ that had the majority of the samples misclassified as being from class $N$ (first column of Table 7). Also, it is important to stress that the accuracy calculated in this work do consider unbalanced datasets [25].

\subsubsection{Three-class problem}

We have also evaluated OPF considering the three-class dataset division proposed by Llamedo and Matínez [15], where classes $F$ and $Q$ are merged into class $V$. Table 8 presents the accuracy results obtained considering the three-class problem. Once again, the best result was obtained with Manhattan distance and feature extractor D (91.42\%), as happened in the five-class problem (Table 5). Although some classes have been merged, we still have an unbalanced dataset. The aggregation of classes $F$ and $Q$ into class $V$ has smoothed such problem, but class $C$ still concentrates approximately $90 \%$ of the samples. Table 9 presents the results obtained in terms of sensitivity, specificity and harmonic mean.

Considering class $N$, Canberra and squared Chi-squared distances together with the feature extractor $\mathrm{C}$ presented the best values for the harmonic mean $(H)(0.78)$. Additionally, squared Chi-squared and the same feature extractor achieved the best result over class $S$. This may indicate that aggregation into 3 classes does not influence the measure $H$ for classes $N$ and $S$, the same values where obtained in the five-class problem (Table 6). In regard to class $V$, the best value $(H=0.88)$ was obtained with Euclidean and Manhattan distances over the feature extractor F. Therefore, the aggregation into three classes seemed to improve the results for the classes $V, F$ and $Q$, which are now clustered into class $V$.

Table 10 presents the OPF computational time (in seconds) for the training and test phases, being the fastest approaches the ones using Bray-Curtis and Manhattan metrics, since they are simpler to compute. It is important to highlight that these results are accompanied by a
Table 8 OPF accuracy considering three classes

\begin{tabular}{|c|c|c|c|c|}
\hline \multirow[t]{2}{*}{ Dataset } & \multicolumn{4}{|c|}{ Distance metric } \\
\hline & \multicolumn{2}{|l|}{ Euclidean $(\%)$} & Chi-square (\%) & Manhattan (\%) \\
\hline A & \multicolumn{2}{|l|}{81.00} & 83.41 & 77.82 \\
\hline B & \multicolumn{2}{|l|}{80.43} & 88.89 & 80.18 \\
\hline $\mathrm{C}$ & \multicolumn{2}{|l|}{81.41} & 87.68 & 84.61 \\
\hline $\mathrm{D}$ & \multicolumn{2}{|l|}{90.92} & 89.13 & 91.42 \\
\hline E & \multicolumn{2}{|l|}{86.81} & 89.08 & 86.80 \\
\hline F & \multicolumn{2}{|l|}{89.46} & 85.35 & 90.78 \\
\hline \multirow[t]{2}{*}{ Dataset } & \multicolumn{4}{|l|}{ Distance metric } \\
\hline & $\begin{array}{l}\text { Canberra } \\
(\%)\end{array}$ & \multicolumn{2}{|c|}{$\begin{array}{l}\text { Squared Chi-squared } \\
(\%)\end{array}$} & $\begin{array}{l}\text { Bray-Curtis } \\
(\%)\end{array}$ \\
\hline A & 78.29 & \multicolumn{2}{|l|}{76.43} & 80.20 \\
\hline B & 81.21 & \multicolumn{2}{|c|}{81.46} & 88.48 \\
\hline $\mathrm{C}$ & 85.18 & \multicolumn{2}{|c|}{82.84} & 76.88 \\
\hline $\mathrm{D}$ & 91.07 & \multicolumn{2}{|l|}{90.94} & 88.92 \\
\hline $\mathrm{E}$ & 86.84 & \multicolumn{2}{|c|}{86.90} & 82.11 \\
\hline $\mathrm{F}$ & 86.87 & \multicolumn{2}{|c|}{85.94} & 78.73 \\
\hline
\end{tabular}

The most accurate result is indicated in bold

satisfactory classification performance, since OPF with Manhattan distance obtained generally very good classification results.

\subsubsection{Comparative analysis of the classifiers considering the five-class problem}

In order to compare the performance of OPF over traditional classifiers (SVM-RBF ${ }^{4}$ and Bayesian classifier), we considered only the two best distance metrics found in the previous section, i.e., Manhattan and squared Chi-squared distances. Therefore, we can summarize the techniques to be compared as follows:

- OPF-L1: OPF with Manhattan distance;

- OPF-SCS: OPF with squared Chi-squared distance;

- SVM-RBF: support vector machines using RBF kernel $;^{5}$

- BC: Bayesian Classifier.

Table 11 shows the accuracy obtained for each feature extractor and classifier considering five classes of heartbeats. The most accurate technique was SVM-RBF with $94.09 \%$ of classification accuracy, followed by OPF-L1, BC and OPF-SCS, which obtained 91.21, 90.95 and $90.75 \%$ of classification accuracies, respectively,

\footnotetext{
${ }^{4}$ SVM parameters were optimized through cross-validation procedure.

${ }^{5}$ SVM implementation used was based on LIBSVM [4].
} 
Table 9 Specificity, sensitivity and their harmonic mean considering the OPF classifier and the three-class categorization

\begin{tabular}{|c|c|c|c|c|}
\hline \multirow[t]{2}{*}{ Metric } & \multirow[t]{2}{*}{ Dataset } & \multicolumn{3}{|l|}{ Heartbeat class } \\
\hline & & $\begin{array}{l}N \\
\mathrm{H}-\mathrm{Se}-\mathrm{Sp}\end{array}$ & $\begin{array}{l}S \\
\mathrm{H}-\mathrm{Se}-\mathrm{Sp}\end{array}$ & $\begin{array}{l}V \\
\mathrm{H}-\mathrm{Se}-\mathrm{Sp}\end{array}$ \\
\hline \multirow[t]{6}{*}{ Euclidean } & $\mathrm{A}$ & $066-085-054$ & $002-001-097$ & $082-077-088$ \\
\hline & $\mathrm{B}$ & $050-086-035$ & $005-002-097$ & $062-047-090$ \\
\hline & $\mathrm{C}$ & $074-085-066$ & $031-018-095$ & $080-072-089$ \\
\hline & $\mathrm{D}$ & $073-096-059$ & $030-018-099$ & $081-070-096$ \\
\hline & $\mathrm{E}$ & $065-092-050$ & $006-003-098$ & $074-060-094$ \\
\hline & $\mathrm{F}$ & $074-093-062$ & $022-012-099$ & $\mathbf{0 8 8}-082-094$ \\
\hline \multirow[t]{6}{*}{ Chi-square } & $\mathrm{A}$ & $016-093-009$ & $003-001-099$ & $016-009-094$ \\
\hline & $\mathrm{B}$ & $002-100-001$ & $000-000-100$ & $002-001-100$ \\
\hline & $\mathrm{C}$ & $015-098-008$ & $002-001-100$ & $016-009-098$ \\
\hline & $\mathrm{D}$ & $004-100-002$ & $000-000-100$ & $004-002-100$ \\
\hline & $\mathrm{E}$ & $004-100-002$ & $000-000-100$ & $004-002-100$ \\
\hline & $\mathrm{F}$ & $012-095-006$ & $002-001-099$ & $011-006-097$ \\
\hline \multirow[t]{6}{*}{ Manhattan } & $\mathrm{A}$ & $066-081-056$ & $003-002-095$ & $083-080-086$ \\
\hline & $\mathrm{B}$ & $046-087-031$ & $006-003-097$ & $056-041-090$ \\
\hline & $\mathrm{C}$ & $076-088-066$ & $031-018-097$ & $081-072-091$ \\
\hline & $\mathrm{D}$ & $071-096-056$ & $023-013-099$ & $081-070-097$ \\
\hline & $\mathrm{E}$ & $064-092-048$ & $006-003-098$ & $073-060-094$ \\
\hline & $\mathrm{F}$ & $072-095-058$ & $011-006-099$ & $\mathbf{0 8 8}-081-096$ \\
\hline \multirow[t]{6}{*}{ Canberra } & A & $071-081-063$ & $047-031-098$ & $077-072-083$ \\
\hline & B & $042-088-028$ & $005-003-098$ & $051-035-091$ \\
\hline & $\mathrm{C}$ & $\mathbf{0 7 8}-088-071$ & $056-039-096$ & $081-073-092$ \\
\hline & $\mathrm{D}$ & $071-096-057$ & $026-015-099$ & $081-070-097$ \\
\hline & $\mathrm{E}$ & $064-092-049$ & $007-004-098$ & $074-061-094$ \\
\hline & $\mathrm{F}$ & $064-092-049$ & $007-004-099$ & $078-068-093$ \\
\hline \multirow[t]{6}{*}{ Squared Chi-square } & A & $066-080-056$ & $006-003-097$ & $078-074-083$ \\
\hline & $\mathrm{B}$ & $048-088-033$ & $004-002-098$ & $059-044-090$ \\
\hline & $\mathrm{C}$ & $\mathbf{0 7 8}-085-072$ & 060-044-095 & $081-074-090$ \\
\hline & $\mathrm{D}$ & $074-096-060$ & $032-019-099$ & $081-070-096$ \\
\hline & $\mathrm{E}$ & $065-093-050$ & $006-003-098$ & $074-061-094$ \\
\hline & $\mathrm{F}$ & $069-090-056$ & $020-011-099$ & $082-074-091$ \\
\hline \multirow[t]{6}{*}{ Bray-Curtis } & $\mathrm{A}$ & $051-086-036$ & $018-010-098$ & $057-042-088$ \\
\hline & $\mathrm{B}$ & $001-099-001$ & $000-000-100$ & $001-000-099$ \\
\hline & $\mathrm{C}$ & $055-083-041$ & $008-004-096$ & $057-042-087$ \\
\hline & $\mathrm{D}$ & $002-100-001$ & $001-000-100$ & $000-000-100$ \\
\hline & $\mathrm{E}$ & $035-090-022$ & $005-003-098$ & $045-030-092$ \\
\hline & $\mathrm{F}$ & $053-085-039$ & $003-002-096$ & $055-039-089$ \\
\hline
\end{tabular}

The best values for the harmonic mean are indicated in bold considering the feature extractor D. Additionally, Table 12 presents the sensitivity, specificity and harmonic mean results.

From Table 12, one can realize that the best results in terms of harmonic mean were obtained for class $N$ with SVM-RBF and feature extractor D $(80.00 \%)$. This result is about $2 \%$ higher than the second best result obtained by OPF-SCS with feature extractor C $(78.00 \%)$. In regard to class $S$, the best classifier was OPF-SCS using feature extractor $\mathrm{C}$, followed by SVM-RBF with $51 \%$ of classification accuracy, which achieved the best recognition rates for classes $V$ and $F$. 
Table 10 OPF computational time (in s) considering the threeclass problem

\begin{tabular}{|c|c|c|c|c|c|c|c|c|c|}
\hline & \multicolumn{9}{|c|}{ Distance metrics } \\
\hline & \multicolumn{3}{|l|}{ Euclidean } & \multicolumn{3}{|c|}{ Chi-square } & \multicolumn{3}{|c|}{ Manhattan } \\
\hline & Training & Test & Total & Training & Test & Total & Training & Test & Total \\
\hline A & 445.18 & 682.89 & 1128.07 & 3230.23 & 1987.14 & 5217.37 & 335.07 & 584.79 & 919.86 \\
\hline B & 145.53 & 173.48 & 319.01 & 416.65 & 6.57 & 423.21 & 54.50 & 94.53 & 149.03 \\
\hline $\mathrm{C}$ & 142.70 & 177.63 & 320.34 & 464.50 & 102.25 & 566.74 & 55.19 & 97.24 & 152.43 \\
\hline $\mathrm{D}$ & 128.90 & 132.96 & 261.86 & 299.52 & 2.74 & 302.26 & 40.09 & 53.07 & 93.15 \\
\hline $\mathrm{E}$ & 172.76 & 181.13 & 353.89 & 650.34 & 14.66 & 665.00 & 80.58 & 108.42 & 189.00 \\
\hline \multirow[t]{4}{*}{$\mathrm{F}$} & 317.86 & 444.96 & 762.82 & 2103.07 & 818.28 & 2921.35 & 219.07 & 360.48 & 579.55 \\
\hline & \multicolumn{9}{|c|}{ Distance metrics } \\
\hline & \multicolumn{3}{|l|}{ Canberra } & \multicolumn{3}{|c|}{ Squared Chi-squared } & \multicolumn{3}{|c|}{ Bray-Curtis } \\
\hline & Training & Test & Total & Training & Test & Total & Training & Test & Total \\
\hline A & 1479.33 & 1588.78 & 3068.11 & 1478.00 & 1588.67 & 3066.67 & 1143.30 & 1168.50 & 2311.81 \\
\hline B & 187.74 & 161.54 & 349.28 & 189.63 & 183.48 & 373.11 & 43.35 & 0.06 & 43.41 \\
\hline $\mathrm{C}$ & 203.89 & 225.74 & 429.63 & 204.11 & 230.33 & 434.44 & 104.88 & 118.07 & 222.96 \\
\hline $\mathrm{D}$ & 130.45 & 142.55 & 273.00 & 132.00 & 136.51 & 268.52 & 35.22 & 6.01 & 41.22 \\
\hline $\mathrm{E}$ & 297.03 & 198.04 & 495.07 & 299.07 & 227.00 & 526.07 & 150.78 & 125.34 & 276.12 \\
\hline $\mathrm{F}$ & 969.35 & 997.24 & 1966.59 & 9682.9 & 976.22 & 1944.50 & 431.96 & 519.69 & 951.66 \\
\hline
\end{tabular}

Best values are indicated in bold
Table 11 Accuracy rates obtained considering AAMI five classes

\begin{tabular}{|c|c|c|c|c|}
\hline \multirow[t]{2}{*}{ Dataset } & \multicolumn{4}{|l|}{ Classifier } \\
\hline & OPF-L1 (\%) & OPF-SCS $(\%)$ & SVM-RBF (\%) & $\mathrm{BC}(\%)$ \\
\hline $\mathrm{A}$ & 77.57 & 76.14 & 88.21 & 80.69 \\
\hline B & 79.43 & 80.61 & 84.06 & 79.52 \\
\hline $\mathrm{C}$ & 84.46 & 82.63 & 89.82 & 81.37 \\
\hline $\mathrm{D}$ & 91.21 & 90.75 & 94.09 & 90.95 \\
\hline $\mathrm{E}$ & 86.47 & 86.62 & 87.06 & 86.82 \\
\hline $\mathrm{F}$ & 90.39 & 85.70 & 87.12 & 89.14 \\
\hline
\end{tabular}

The best accuracy value is indicated in bold

Table 13 displays the mean execution times considering the training, testing and total time (training + testing) required by each classifier. ${ }^{6}$ The fastest classifier in the training phase was the $\mathrm{BC}$ in all datasets, followed by OPFL1. The OPF-SCS was faster than SVM-RBF in all datasets as well, except for dataset F, where SVM-RBF had the third best time. The excessive times of SVM-RBF were due to the grid search that is necessary to fine-tune its parameters.

In the test phase, the best computational time was obtained by SVM-RBF (6.7 s), being almost 8 times faster than OPF-L1 (53.3 s), both with feature extractor D. The third fastest technique was OPF-SCS (131.3 s), while BC, despite being the fastest in the training phase, took $173 \mathrm{~s}$ to

\footnotetext{
${ }^{6}$ We have executed all techniques 10 times for statistical purposes.
}

classify the samples. In resume, SVM-RBF was the fastest in the classification phase, followed by OPF-L1, OPF-SCS and BC. Usually, SVM is fast for classifying samples, since it only considers the support vectors for such purpose, while OPF may need to evaluate a considerable number of training samples for that. However, if we consider the total time, OPF-L1 was the most efficient technique, which may lead us to consider it as a very suitable classifier concerning the trade-off between low computational time and high recognition rate.

Table 14 presents the confusion matrix related to SVM-RBF classifier in the five-class problem for the Dataset A [5]. It can be noted a confusion of class SVEB with class $N$, where only 37 (2\%) samples were classified correctly for class SVEB. However, using the OPF-SCS classifier with Dataset C [29], the amount of samples correctly classified in the same class was around $43 \%$. Thus, to detect Cardiac arrhythmia, also known as cardiac dysrhythmia or irregular heartbeat, the accuracy over class SVEB is usually considered most important. As such, the OPF-SCS accuracy obtained for this class, which is much higher than the one of SVM-RBF, is of greater clinical relevance.

\subsubsection{Comparative analysis of the classifiers considering the three-class problem}

In this section, we analyze the performance and computational time of all classifiers considering the three-class 
Table 12 Harmonic mean, specificity and sensitivity obtained considering five classes and all classifiers

\begin{tabular}{|c|c|c|c|c|c|c|}
\hline \multirow[t]{2}{*}{ Metric } & \multirow[t]{2}{*}{ Dataset } & \multicolumn{5}{|l|}{ Heartbeat class } \\
\hline & & $\begin{array}{l}N \\
\mathrm{H}-\mathrm{Se}-\mathrm{Sp}\end{array}$ & $\begin{array}{l}S \\
\mathrm{H}-\mathrm{Se}-\mathrm{Sp}\end{array}$ & $\begin{array}{l}V \\
\mathrm{H}-\mathrm{Se}-\mathrm{Sp}\end{array}$ & $\begin{array}{l}F \\
\mathrm{H}-\mathrm{Se}-\mathrm{Sp}\end{array}$ & $\begin{array}{l}Q \\
\mathrm{H}-\mathrm{Se}-\mathrm{Sp}\end{array}$ \\
\hline \multirow[t]{6}{*}{ OPF-L1 } & A & $066-081-056$ & $003-002-095$ & $086-082-090$ & $051-035-096$ & $000-000-100$ \\
\hline & B & $046-087-031$ & $006-003-097$ & $051-035-090$ & $001-000-099$ & $000-000-100$ \\
\hline & $\mathrm{C}$ & $076-088-066$ & $031-018-097$ & $085-078-093$ & $013-007-098$ & $000-000-100$ \\
\hline & $\mathrm{D}$ & $071-096-056$ & $023-013-099$ & $085-075-098$ & $008-004-099$ & $000-000-100$ \\
\hline & $\mathrm{E}$ & $064-092-048$ & $006-003-098$ & $075-061-097$ & $023-013-097$ & $000-000-100$ \\
\hline & $\mathrm{F}$ & $072-095-058$ & $010-006-099$ & $090-083-098$ & $034-021-098$ & $000-000-100$ \\
\hline \multirow[t]{6}{*}{ OPF-SCS } & A & $065-080-056$ & $006-003-097$ & $081-076-086$ & $037-023-097$ & $000-000-100$ \\
\hline & B & $048-088-033$ & $004-002-098$ & $053-037-091$ & $001-001-098$ & $000-000-100$ \\
\hline & $\mathrm{C}$ & $078-085-072$ & 060-044-095 & $085-078-093$ & $022-012-097$ & $000-000-100$ \\
\hline & $\mathrm{D}$ & $073-096-060$ & $032-019-099$ & $085-075-097$ & $009-005-099$ & $000-000-100$ \\
\hline & $\mathrm{E}$ & $065-092-050$ & $006-003-098$ & $076-062-097$ & $027-016-097$ & $000-000-100$ \\
\hline & $\mathrm{F}$ & $069-090-056$ & $020-011-099$ & 087-079-098 & $008-004-093$ & $000-000-100$ \\
\hline \multirow[t]{6}{*}{ SVM-RBF } & A & $074-092-063$ & $006-003-099$ & $093-091-096$ & $\mathbf{0 8 2}-072-097$ & $000-000-100$ \\
\hline & B & $049-091-033$ & $001-000-100$ & $061-045-093$ & $000-000-098$ & $\mathbf{0 0 8}-005-100$ \\
\hline & $\mathrm{C}$ & $070-094-056$ & $015-008-098$ & $089-083-097$ & $003-002-100$ & $000-000-100$ \\
\hline & $\mathrm{D}$ & $\mathbf{0 8 0}-098-067$ & $051-035-099$ & $090-082-099$ & $004-002-100$ & $000-000-100$ \\
\hline & $\mathrm{E}$ & $070-092-057$ & $012-007-099$ & $089-082-098$ & $001-001-094$ & $000-000-100$ \\
\hline & $\mathrm{F}$ & $074-091-063$ & $026-015-099$ & $\mathbf{0 9 3}-089-096$ & $030-018-096$ & $000-000-100$ \\
\hline \multirow[t]{6}{*}{$\mathrm{BC}$} & A & $066-084-054$ & $002-001-097$ & $084-078-091$ & $055-038-097$ & $000-000-100$ \\
\hline & B & $050-086-035$ & $005-002-097$ & $056-041-090$ & $001-001-099$ & $000-000-100$ \\
\hline & $\mathrm{C}$ & $074-085-066$ & $031-018-095$ & $084-078-091$ & $013-007-097$ & $000-000-100$ \\
\hline & $\mathrm{D}$ & $073-096-059$ & $029-017-099$ & 085-076-097 & $007-004-099$ & $000-000-100$ \\
\hline & $\mathrm{E}$ & $064-093-049$ & $005-003-098$ & $076-062-097$ & $027-016-097$ & $000-000-100$ \\
\hline & $\mathrm{F}$ & $074-093-062$ & $021-012-099$ & $091-086-097$ & $031-018-097$ & $000-000-100$ \\
\hline
\end{tabular}

The best values are indicated in bold

division proposed by [15]. Table 15 presents the recognition rates for each pair classifier/feature extractor method, being the sensitivity, specificity and harmonic mean results displayed in Table 16.

In regard to class $N$, the SVM-RBF classifier has obtained the best harmonic mean value with feature extractor D; meanwhile, OPF-SCS was the most accurate technique for class $S$ using feature extractor $\mathrm{C}$. These results are consistent with those obtained considering five classes. With respect to class $V$, three classifiers obtained the best harmonic mean values: SVM-RBC with feature extractor A, and OPF-L1 and BC with feature extractor F. However, in all these three cases, the values are followed by low sensitivity values for class $S$.

Table 17 presents the mean computational time in seconds concerning all techniques. Once again, the lowest computational time for training was achieved by BC and followed by OPF-L1 for all datasets. Except for feature extractors $\mathrm{C}$ and $\mathrm{F}$, where OPF took longer to train, SVM$\mathrm{RBF}$ classifier was the most costly technique for training the samples. Relatively to the five-class problem, similar computational times could be observed for BC and OPFbased classifiers, evidencing the robustness of these classifiers when dealing with different number of classes. As expected, the SVM computational time decreased, since we have less classes to be analyzed during the pair-wise comparison against them. ${ }^{7}$ Last but not least, SVM-RBF was the fastest technique for the classification phase, while OPF-L1 obtained the lowest execution time considering both training and test phases.

Also, based on a similar analysis to the one carried out with the data in Table 14, it could be confirmed that also in the three-class problem, OPF-SCS is the most appropriate to identify the pathological classes, i.e., the ones with greater clinical interest. Luz et al. [17] considered only the Euclidean metric and obtaining highest accuracy rates of 90.7 and $90.9 \%$ in the 3- and 5-class problems,

\footnotetext{
${ }^{7}$ LIBSVM implements the one-against-one method for multi-class tasks.
} 
Table 13 Mean computational time (in s) required in the AAMI five-class problem

\begin{tabular}{|c|c|c|c|c|c|c|}
\hline \multirow[t]{3}{*}{ Dataset } & \multicolumn{6}{|l|}{ Classifier } \\
\hline & \multicolumn{3}{|l|}{ OPF-L1 } & \multicolumn{3}{|l|}{ OPF-SCS } \\
\hline & Train & Test & Total & Train & Test & Total \\
\hline A & 337.0 (1.6) & $584.2(0.7)$ & $921.2(1.8)$ & $1487.2(10.8)$ & $1604.7(12.4)$ & $3091.9(22.3)$ \\
\hline B & $54.8(0.7)$ & $102.9(13.4)$ & 157.7 (13.7) & $191.9(2.4)$ & $181.2(4.0)$ & 373.1 (3.6) \\
\hline $\mathrm{C}$ & $55.5(0.4)$ & $95.1(4.0)$ & $150.6(3.9)$ & $206.9(2.0)$ & 242.5 (7.9) & 449.4 (9.9) \\
\hline $\mathrm{D}$ & $40.3(0.2)$ & $53.3(7.3)$ & $93.6(7.2)$ & $132.4(0.8)$ & $131.3(4.9)$ & $263.7(5.4)$ \\
\hline $\mathrm{E}$ & $81.1(0.8)$ & $115.1(3.5)$ & $196.2(3.2)$ & $302.2(3.7)$ & $223.8(4.9)$ & $525.9(6.9)$ \\
\hline $\mathrm{F}$ & $220.4(2.1)$ & $380.3(6.9)$ & $600.8(6.1)$ & $974.9(6.7)$ & $990.0(3.4)$ & $1964.8(9.9)$ \\
\hline \multirow[t]{3}{*}{ Dataset } & \multicolumn{3}{|l|}{ Classifier } & & & \\
\hline & \multicolumn{3}{|l|}{ SVM-RBF } & \multicolumn{3}{|l|}{$\mathrm{BC}$} \\
\hline & Train & Test & Total & Train & Test & Total \\
\hline A & $2668.4(26.2)$ & $32.2(6.7)$ & $2700.6(31.3)$ & $62.9(0.3)$ & $1622.2(8.6)$ & $1685.2(8.9)$ \\
\hline B & $576.0(474.0)$ & $12.6(1.7)$ & $588.6(472.3)$ & $11.1(0.2)$ & $236.0(2.7)$ & $247.1(2.8)$ \\
\hline $\mathrm{C}$ & $195.3(8.6)$ & $6.9(2.1)$ & $202.2(10.5)$ & $11.9(0.0)$ & $253.8(3.0)$ & 265.7 (2.9) \\
\hline $\mathrm{D}$ & $170.7(5.7)$ & $6.7(0.0)$ & $177.4(5.7)$ & $8.7(0.1)$ & $173.0(1.9)$ & 181.7 (1.9) \\
\hline $\mathrm{E}$ & $546.5(48.2)$ & $9.4(0.7)$ & $555.9(47.5)$ & $15.6(0.1)$ & $354.1(4.1)$ & $369.6(4.0)$ \\
\hline $\mathrm{F}$ & $608.7(9.5)$ & $15.3(0.1)$ & $624.0(9.6)$ & $42.0(0.1)$ & $1058.8(9.2)$ & $1100.8(9.2)$ \\
\hline
\end{tabular}

The standard deviation is also displayed. The lowest times are indicated in bold
Table 14 Confusion matrices obtained for SVM-RBF and OPF-SCS classifiers

\begin{tabular}{|c|c|c|c|c|c|}
\hline & \multicolumn{3}{|c|}{ True class } & \multirow[b]{2}{*}{$F$} & \multirow[b]{2}{*}{$Q$} \\
\hline & $N$ & SVEB & VEB & & \\
\hline \multicolumn{5}{|c|}{ SVM-RBF-Dataset A [5] } & \\
\hline \multicolumn{5}{|c|}{ Classified as } & \\
\hline$N$ & 40099 & 1705 & 221 & 83 & 3 \\
\hline SVEB & 361 & 37 & 3 & 0 & 0 \\
\hline VEB & 2285 & 23 & 2933 & 15 & 4 \\
\hline$F$ & 1436 & 21 & 61 & 290 & 0 \\
\hline$Q$ & 0 & 0 & 0 & 0 & 0 \\
\hline \multicolumn{5}{|c|}{ OPF-SCS-Dataset C [29] } & \\
\hline \multicolumn{6}{|c|}{ Classified as } \\
\hline$N$ & 37677 & 612 & 591 & 322 & 2 \\
\hline SVEB & 2495 & 802 & 33 & 2 & 0 \\
\hline VEB & 2798 & 412 & 2514 & 17 & 5 \\
\hline$F$ & 1245 & 9 & 81 & 47 & 0 \\
\hline$Q$ & 3 & 2 & 0 & 0 & 0 \\
\hline
\end{tabular}

respectively, considering in both cases the extraction method proposed by [33]. However, the present work could improve the accuracy of OPF with Manhattan distance,
Table 15 Classification accuracy considering the three-class problem

\begin{tabular}{|c|c|c|c|c|}
\hline \multirow[t]{2}{*}{ Dataset } & \multicolumn{4}{|l|}{ Classifier } \\
\hline & OPF-L1 & OPF-SCS & SVM-RBF & $\mathrm{BC}$ \\
\hline A & 77.82 & 76.43 & 80.01 & 80.98 \\
\hline B & 80.18 & 81.46 & 84.29 & 80.31 \\
\hline C & 84.61 & 82.84 & 90.01 & 81.53 \\
\hline $\mathrm{D}$ & 91.42 & 90.94 & 93.72 & 91.17 \\
\hline $\mathrm{E}$ & 86.80 & 86.90 & 88.45 & 87.07 \\
\hline F & 90.78 & 85.94 & 83.66 & 89.47 \\
\hline
\end{tabular}

The best accuracy value is indicated in bold

obtaining 91.42 and $91.21 \%$ in the 3- and 5-class problems, respectively, for the same dataset and with computational time inferior to the one achieved by Luz et al. [17]. This considerable increase in accuracy directly leads to a more accurate detection of pathological classes. As such, it is possible to identify more precisely a cardiac arrhythmia with the Manhattan distance than with Euclidean one. Again, it should be stressed that the aforementioned classes are of great importance for clinical analysis, and that the SVM classifier could not detect accurately enough the samples of these classes. 
Table 16 Harmonic mean, specificity and sensitivity considering three classes and all classifiers

\begin{tabular}{|c|c|c|c|c|}
\hline \multirow[t]{2}{*}{ Metric } & \multirow[t]{2}{*}{ Dataset } & \multicolumn{3}{|l|}{ Heartbeat class } \\
\hline & & $\begin{array}{l}N \\
\mathrm{H}-\mathrm{Se}-\mathrm{Sp}\end{array}$ & $\begin{array}{l}S \\
\mathrm{H}-\mathrm{Se}-\mathrm{Sp}\end{array}$ & $\begin{array}{l}V \\
\mathrm{H}-\mathrm{Se}-\mathrm{Sp}\end{array}$ \\
\hline \multirow[t]{6}{*}{ OPF-L1 } & A & $066-081-056$ & $003-002-095$ & $083-080-086$ \\
\hline & B & $046-087-031$ & $006-003-097$ & $056-041-090$ \\
\hline & $\mathrm{C}$ & $076-088-066$ & $031-018-097$ & $081-072-091$ \\
\hline & $\mathrm{D}$ & $071-096-056$ & $023-013-099$ & $081-070-097$ \\
\hline & $\mathrm{E}$ & $064-092-048$ & $006-003-098$ & $073-060-094$ \\
\hline & $\mathrm{F}$ & $072-095-058$ & $011-006-099$ & $\mathbf{0 8 8}-081-096$ \\
\hline \multirow[t]{6}{*}{ OPF-SCS } & A & $066-080-56$ & $006-003-097$ & $078-074-083$ \\
\hline & B & $048-088-033$ & $004-002-098$ & $059-044-090$ \\
\hline & $\mathrm{C}$ & $078-085-072$ & 060-044-095 & $081-074-090$ \\
\hline & $\mathrm{D}$ & $074-096-060$ & $032-019-099$ & $081-070-096$ \\
\hline & $\mathrm{E}$ & $065-093-050$ & $006-003-098$ & $074-061-094$ \\
\hline & $\mathrm{F}$ & $069-090-056$ & $020-011-099$ & $082-074-091$ \\
\hline \multirow[t]{6}{*}{ SVM-RBF } & A & $072-082-065$ & $007-004-098$ & $\mathbf{0 8 8}-092-084$ \\
\hline & B & $053-090-038$ & $001-000-100$ & $069-056-091$ \\
\hline & $\mathrm{C}$ & $070-095-056$ & 012-007-099 & $083-074-095$ \\
\hline & $\mathrm{D}$ & $\mathbf{0 8 0}-098-067$ & $052-035-099$ & $084-074-098$ \\
\hline & $\mathrm{E}$ & $071-093-057$ & $012-006-099$ & $086-080-093$ \\
\hline & $\mathrm{F}$ & $072-087-062$ & $023-013-099$ & $086-085-088$ \\
\hline \multirow[t]{6}{*}{$\mathrm{BC}$} & A & $066-085-054$ & $002-001-097$ & $082-077-088$ \\
\hline & B & $050-086-035$ & $005-002-097$ & $062-047-089$ \\
\hline & $\mathrm{C}$ & $074-085-066$ & $031-018-095$ & $080-072-089$ \\
\hline & $\mathrm{D}$ & $073-096-059$ & $029-017-099$ & $082-071-096$ \\
\hline & $\mathrm{E}$ & $065-093-049$ & $005-003-098$ & $074-060-094$ \\
\hline & $\mathrm{F}$ & $074-093-062$ & $021-012-099$ & $\mathbf{0 8 8}-083-094$ \\
\hline
\end{tabular}

The best values are indicated in bold

\section{Conclusions and future works}

In this paper, a detailed study about the performance and computational time of supervised classification algorithms regarding the task of arrhythmia detection in ECG signals was presented. The main contributions of this work are: (1) to evaluate the OPF classifier in the task of arrhythmia detection, (2) to evaluate six distances with OPF, among which the best accuracy rates were obtained by the Manhattan metric, while better generalization (i.e., the accuracy achieved per class) was attained using squared Chi-square distance, (3) to test six feature extraction techniques and investigate which one leads to better recognition rates and generalization, (4) to compare OPF against support vector machines and a Bayesian classifier, being found that OPF was the less generalist, while the SVM classifier was the most accurate, and, finally, (5) to find that OPF achieved the best trade-off between computational load and recognition rate.

OPF being less generalist with respect to classes $V$ and $S$, which are of great clinical significance regarding class $N$, one can conclude that this classifier is more appropriate for the classification of arrhythmias in ECG signals than the SVM and Bayesian classifiers.

Since we observed that OPF and SVM-RBF were the most accurate classifiers, our future works will be guided to explore the synergy between these classifiers in order to build an ensemble of classifiers aiming at increasing the 
Table 17 Mean computational time (in s) considering the threeclass problem

\begin{tabular}{|c|c|c|c|c|c|c|}
\hline \multirow[t]{3}{*}{ Dataset } & \multicolumn{6}{|l|}{ Classifier } \\
\hline & \multicolumn{3}{|l|}{ OPF-L1 } & \multicolumn{3}{|l|}{ OPF-SCS } \\
\hline & Train & Test & Total & Train & Test & Total \\
\hline A & $336.0(1.7)$ & $575.2(11.5)$ & $911.2(9.9)$ & $1486.1(7.1)$ & $1597.9(8.1)$ & $3084.1(15.1)$ \\
\hline B & $54.5(0.2)$ & $93.0(2.8)$ & $147.5(3.0)$ & $191.2(1.7)$ & $192.3(7.8)$ & $383.4(9.4)$ \\
\hline $\mathrm{C}$ & $55.5(0.3)$ & $92.3(9.2)$ & $147.7(9.3)$ & $206.2(2.0)$ & 233.5 (12.7) & 439.7 (14.1) \\
\hline $\mathrm{D}$ & $40.2(0.2)$ & $52.2(5.2)$ & $92.4(5.4)$ & $132.6(0.5)$ & $134.1(3.8)$ & $266.7(3.5)$ \\
\hline $\mathrm{E}$ & $81.0(0.4)$ & $105.9(4.2)$ & $186.9(4.0)$ & $301.6(2.6)$ & $224.8(4.2)$ & $526.4(2.2)$ \\
\hline $\mathrm{F}$ & 221.7 (2.7) & 368.8 (11.6) & $590.5(14.1)$ & $973.4(4.7)$ & $977.3(2.8)$ & $1950.7(6.8)$ \\
\hline \multirow[t]{3}{*}{ Dataset } & \multicolumn{3}{|l|}{ Classifier } & & & \\
\hline & \multicolumn{3}{|l|}{ SVM-RBF } & \multicolumn{3}{|l|}{$\mathrm{BC}$} \\
\hline & Train & Test & Total & Train & Test & Total \\
\hline A & $2069.7(23.7)$ & $25.9(5.4)$ & $2095.6(28.6)$ & $62.5(0.1)$ & $971.2(5.2)$ & $1033.7(5.3)$ \\
\hline B & $280.5(2.9)$ & $11.6(0.7)$ & $292.2(3.6)$ & $11.0(0.1)$ & $141.5(0.7)$ & $152.5(0.7)$ \\
\hline $\mathrm{C}$ & $194.8(2.7)$ & $7.7(0.0)$ & $202.5(2.7)$ & $11.7(0.1)$ & $152.9(0.3)$ & $164.6(0.4)$ \\
\hline $\mathrm{D}$ & $165.9(1.0)$ & $6.0(0.0)$ & $171.9(0.9)$ & $8.7(0.1)$ & $105.4(1.4)$ & $114.1(1.5)$ \\
\hline $\mathrm{E}$ & $536.1(58.9)$ & $9.1(0.8)$ & $545.2(58.1)$ & $15.5(0.1)$ & $213.5(2.2)$ & $229.0(2.3)$ \\
\hline $\mathrm{F}$ & $553.3(26.2)$ & $17.0(6.8)$ & $570.3(32.9)$ & $41.6(0.3)$ & $638.5(5.1)$ & $680.1(5.3)$ \\
\hline
\end{tabular}

The standard deviation is also displayed. The lowest times are indicated in bold recognition rate of arrhythmia detection in ECG signals, as well as to evaluate other traditional and most recent feature extraction methods.

Acknowledgments The first author thanks the Brazilian National Council for Research and Development $(\mathrm{CNPq})$ for providing financial support through Grants \# 470501/2013-8 and \# 301928/2014-2.

The sixth author is grateful to CNPq Grants \#306166/2014-3 and \#470571/2013-6, as well as to São Paulo Research Foundation (FAPESP) Grant \#2014/16250-9.

The last author gratefully acknowledges the funding of Project NORTE-01-0145-FEDER-000022-SciTech-Science and Technology for Competitive and Sustainable Industries cofinanced by "Programa Operacional Regional do Norte (NORTE2020)" through "Fundo Europeu de Desenvolvimento Regional (FEDER)".

\section{References}

1. Abawajy JH, Kelarev AV, Chowdhury M (2013) Multistage approach for clustering and classification of ECG data. Comput Methods Progr Biomed 112:720-730

2. Allène C, Audibert JY, Couprie M, Keriven R (2010) Some links between extremum spanning forests, watersheds and min-cuts. Image Vis Comput 28(10):1460-1471

3. ANSI/AAMI (2008) Testing and reporting performance results of cardiac rhythm and ST segment measurement algorithms. Association for the Advancement of Medical Instrumentation -AAMI / American National Standards Institute, Inc.-ANSI. ANSI/AAMI/ ISO EC57, 1998-(R)2008

4. Chang CC, Lin CJ (2011) LIBSVM: a library for support vector machines. ACM Trans Intell Syst Technol 2:1-27. http://www. csie.ntu.edu.tw/ cjlin/libsvm
5. Chazal P, O'Dwyer M, Reilly RB (2004) Automatic classification of heartbeats using ECG morphology and heartbeat interval features. IEEE Trans Biomed Eng 51(7):1196-1206

6. Chen Y, Yu S (2012) Selection of effective features for ECG beat recognition based on nonlinear correlations. Artif Intell Med $54: 43-52$

7. Daamouche A, Hamami L, Alajlan N, Melgani F (2012) A wavelet optimization approach for ECG signal classification. Biomed Signal Process Control 7(4):342-349

8. de Lannoy G, François D, Delbeke J, Verleysen M (2010) Weighted SVMs and feature relevance assessment in supervised heart beat classification. In: Biomedical engineering systems and technologies (BIOSTEC), pp 212-223

9. Dutta S, Chatterjee A, Munshi S (2010) Correlation technique and least square support vector machine combine for frequency domain based ECG beat classification. Med Eng Phys 32(10):1161-1169

10. Güler I, Übeyli ED (2005) ECG beat classifier designed by combined neural network model. Pattern Recognit 38(2):199-208

11. Homaeinezhad MR, Atyabi SA, Tavakkoli E, Toosi HN, Ghaffari A, Ebrahimpour R (2012) ECG arrhythmia recognition via a neuro-SVM-KNN hybrid classifier with virtual QRS image-based geometrical features. Expert Syst Appl 39:2047-2058

12. Ince T, Kiranyaz S, Gabbouj M (2009) A generic and robust system for automated patient-specific classification of ECG signals. IEEE Trans Biomed Eng 56(5):1415-1427

13. Khazaee A, Ebrahimzadeh A (2010) Classification of electrocardiogram signals with support vector machines and genetic algorithms using power spectral features. Biomed Signal Process Control 5(4):252-263

14. Korïek M, Dogan B (2010) ECG beat classificartion using particle swarm optimization and radial basis function neural network. Expert Syst Appl 37:7563-7569

15. Llamedo M, Martínez JP (2011) Heartbeat classification using feature selection driven by database generalization criteria. IEEE Trans Biomed Eng 58(3):616-625 
16. Luz E, Menotti D (2011) How the choice of samples for building arrhythmia classifiers impact their performances. In: Engineering in medicine and biology society (EMBC), annual international conference of the IEEEIEEE, Boston, EUA, pp 4988-4991

17. Luz EJS, Nunes TM, Albuquerque VHC, Papa JP, Menotti D (2013) ECG arrhythmia classification based on optimum-path forest. Expert Syst Appl 40(9):3561-3573

18. Mar T, Zaunseder S, Martínez JP, Llamedo M, Poll R (2011) Optimization of ECG classification by means of feature selection. IEEE Trans Biomed Eng 58(8):2168-2177

19. Mark RG, Schluter PS, Moody GB, Devlin PH, Chernoff D (1982) An annotated ECG database for evaluating arrhythmia detectors. IEEE Trans Biomed Eng 29(8):600

20. Martis RJ, Acharya UR, Mandana K, Ray A, Chakraborty C (2012) Application of principal component analysis to ECG signals for automated diagnosis of cardiac health. Expert Syst Appl 39:11792-11800

21. Martis RJ, Acharya R, Adeli H (2014) Current methods in electrocardiogram characterization. Comput Biol Med 48:133-149

22. Moody GB, Mark RG (2001) The impact of the MIT-BIH arrhythmia database. IEEE Eng Med Biol Mag 20(3):45-50

23. Nejadgholi I, Mohammad MH, Abdolali F (2011) Using phase space reconstruction for patient independent heartbeat classification in comparison with some benchmark methods. Comput Biol Med 41:411-419

24. Papa JP, Falcão AX, Suzuki CTN (2009) LibOPF: a library for the design of optimum-path forest classifiers. Campinas, SP. version 2.1. http://www.ic.unicamp.br/ afalcao/LibOPF

25. Papa JP, Falcão AX, Suzuki CTN (2009) Supervised pattern classification based on optimum-path forest. Int J Imaging Syst Technol 19(2):120-131
26. Papa JP, Falcão AX, de Albuquerque VHC, Tavares JMRS (2012) Efficient supervised optimum-path forest classification for large datasets. Pattern Recognit 45(1):512-520

27. Park KS, Cho BH, Lee DH, Song SH, Lee JS, Chee YJ, Kim IY, Kim SI (2008) Hierarchical support vector machine based heartbeat classification using higher order statistics and hermite basis function. Comput Cardiol 35:229-232

28. Rai HM, Trivedi A, Shukla S (2013) Ecg signal processing for abnormalities detection using multi-resolution wavelet transform and artificial neural network classifier. Measurement 46:3238-3246

29. Song MH, Lee J, Cho SP, Lee KJ, Yoo SK (2005) Support vector machine based arrhythmia classification using reduced features. Int J Control Autom Syst 3(4):509-654

30. Wang JS, Chiang WC, Hsu YL, Yang YTC (2013) ECG arrhythmia classification using a probabilistic neural network with a feature reduction method. Neurocomputing 116:38-45

31. Ye C, Coimbra MT, Kumar BVKV (2010) Arrhythmia detection and classification using morphological and dynamic features of ECG signals. In: IEEE international conference on engineering in medicine and biology society, pp 1918-1921. IEEE, Buenos Aires, Argentina

32. Yu S, Chen Y (2007) Electrocardiogram beat classification based on wavelet transformation and probabilistic neural network. Pattern Recognit Lett 28(10):1142-1150

33. Yu S, Chou K (2008) Integration of independent component analysis and neural networks for ECG beat classification. Expert Syst Appl 34(4):2841-2846 